\title{
Cadaveric study of deep temporal fascia for autologous rhinoplasty grafts: Dimensions of the temporal compartment in Asians
}

\author{
Daekwan $\mathrm{Chi}^{1}$, Jae-Hee Kim ${ }^{1}$, Taek-Kyun Kim ${ }^{1}$, Jae Yong Jeong ${ }^{1}$, Chungmin Shin ${ }^{2}$, Sunje Kim², \\ Sang-Ha $\mathrm{Oh}^{2,3}$ \\ ${ }^{1}$ The PLUS Plastic Surgery, Seoul; ${ }^{2}$ Department of Plastic and Reconstructive Surgery, Chungnam National University Hospital, Daejeon; \\ ${ }^{3}$ Brain Research Institute, Chungnam National University College of Medicine, Daejeon, Korea
}

Background Due to the anatomical complexity of the deep temporal fascia (DTF), practical guidelines for its safe harvest are lacking. However, since the upper temporal compartment (UTC) contains no vital structures, it may provide safe access for DTF harvest. This study aimed to identify the anatomical structures of the temporal compartment in Asian cadavers and to measure their dimensions to enable safe DTF harvest.

Methods The anatomical structures surrounding the temporal compartment were identified in 27 hemifaces from 15 Korean cadavers. After dissection, digital images were acquired and craniometric landmarks were placed upon them to identify the boundaries of the temporal compartment. The horizontal and vertical lengths of the temporal compartment were measured and their surface areas were computationally assessed. Subsequently, differences in the results by sex were evaluated.

Results The five-layer anatomical structure of the UTC was clearly visualized. The UTC was bounded by the temporal septa superiorly and inferiorly, the innominate fascia laterally, and the DTF medially. No vital structures were present within the UTC. The vertical and horizontal lengths of the UTC were $6.41 \pm 0.67 \mathrm{~cm}$ and $10.44 \pm 0.83 \mathrm{~cm}$, respectively, and the surface area of the UTC was $48.52 \pm 5.65 \mathrm{~cm}^{2}$. No statistically significant differences were observed in any dimensions between male and female patients.

Conclusions During rhinoplasty, DTF can be harvested as an autologous graft material from the UTC. An anatomical understanding of the UTC will aid in the safe and simple harvest of a sufficient amount of DTF.

Keywords Fascia / Rhinoplasty / Cadaver
Correspondence: Sunje Kim Department of Plastic and Reconstructive Surgery, Chungnam National University Hospital, 282 Munhwa-ro, Jung-gu, Daejeon 35015, Korea

Tel: +82-42-280-7381

Fax: +82-42-280-7384

E-mail: ksj9243@gmail.com

Sang-Ha Oh

Department of Plastic and Reconstructive Surgery, Chungnam National University Hospital, 282 Munhwa-ro, Jung-gu, Daejeon 35015, Korea

Tel: +82-42-280-7387

Fax: +82-42-280-7384

E-mail: djplastic@cnu.ac.kr

This work was supported by the Chungnam National University Research Fund and the Basic Science Research Program through the National Research Foundation of Korea funded by the Ministry of Science, ICT \& Future Planning (NRF-2018R1A2B6007425) and the Center for Women in Science, Engineering and Technology (WISET) grant funded by the Ministry of Science and ICT (MSIT) under the Program for Returners into R\&D. 


\section{INTRODUCTION}

In rhinoplasty, the deep temporal fascia (DTF) is among the most important autologous graft materials used for various purposes. The DTF is generally utilized alone or as a fascia-wrapped cartilage graft in soft tissue reinforcement in reconstructive rhinoplasty $[1,2]$ and for radix augmentation, correcting dorsal skin irregularities, and reinforcing the tip skin in aesthetic rhinoplasty [3-5]. Since the DTF is harvested from the same surgical field as rhinoplasty and features lower donor site morbidity rates, the DTF has advantages over other autologous tissues such as dermofat tissue [6], tensor fascia lata [7], and mastoid fascia [8]. However, it also has disadvantages, including handling difficulties, partial absorption, donor site alopecia, discomfort due to muscle damage, and possible hematoma [9]. Furthermore, rhinoplasty surgeons are often reluctant to access the temporal area to harvest the DTF owing to its anatomical complexity. The temporal compartment consists of upper and lower parts, the former of which contains no vital structures [10-12]. Therefore, we hypothesized that the upper temporal compartment (UTC) could provide a safe space for DTF harvest. Based on this concept, this study aimed to identify the anatomical structures of the UTC and to measure its dimensions.

\section{METHODS}

This study was approved by the Research Ethics Board of the Chungnam National University Hospital (IRB No. 2019-03042). From March 2019 to March 2020, 27 hemifaces (15 right, 12 left) from 15 Korean cadavers (eight males, seven females; mean age, 65 years) were used to identify the anatomical structures surrounding the temporal compartment. Three hemifaces in which it was difficult to identify landmarks were excluded prior to the study. The nomenclature of the layers and ligamentous structures followed those of a previous report by O'Brien et al. [11]. Two plastic surgeons (JHK and SJK) performed all dissections. Digital images were taken to measure the dimensions of the temporal compartments after full exposure.

\section{Dissection}

After palpation of the superior temporal ridge, an inverted Ushaped skin incision was made from the lateral brow to the mastoid area and the dissection continued from the superior temporal ridge to the upper border of the zygomatic arch in a layer-bylayer fashion.

\section{Landmark identification and lengths and surface areas of the temporal compartments}

Considering the difficulty of directly measuring the surface area in cadavers, indirect photogrammetric measurements were performed. To obtain more objective measurements, we chose craniometric landmarks and semi-landmarks in the temporal area as described in previous studies (Table 1, Fig. 1) [13,14]. Two observers separately placed landmarks on each cadaver image and measured the dimensions of the temporal compartment using Digimizer version 3.1.2.0 software (MedCalc, Mariakerke, Belgium). The vertical and horizontal lengths of the entire temporal compartment (ETC) and the UTC were measured. Subsequently, the surface area of the ETC, UTC, and lower temporal compartment (LTC) were computationally assessed.

Table 1. Definition of craniometric landmarks and semi-landmarks

\begin{tabular}{|c|c|c|c|}
\hline No. & Type & Landmarks $^{\text {a) }}$ & Brief definition \\
\hline 1 & Landmark & Stephanion & The point at which the inferior temporal line crosses the coronal suture \\
\hline 2 & Landmark & Frontotemporale & $\begin{array}{l}\text { The point located generally forward and inward on the superior temporal line directly above the } \\
\text { zygomatic process of the frontal bone }\end{array}$ \\
\hline 3 & Landmark & Marginal process lateral & The most posterior portion of marginal process, taken on the lateral surface of the zygomatic bone \\
\hline 4 & Landmark & Jugale & The deepest point in the curvature of the frontal and temporal malar processes \\
\hline 5 & Landmark & Radiculare & The point on the lateral aspect of the root of the zygomatic process at the deepest incurvature \\
\hline 6 & Semi-landmark & Otobasion superius & The highest point of attachment of the external ear to the scalp \\
\hline 7 & Semi-landmark & SM point & The most laterally positioned point on the supra-mastoid crest \\
\hline 8 & Semi-landmark & P point & $\begin{array}{l}\text { The posterior point of the temporal fossa or the superior temporal line perpendicular to the Frankfurt } \\
\text { horizontal line }\end{array}$ \\
\hline 9 & Semi-landmark & SP point & $\begin{array}{l}\text { The point between the superior point of the superior temporal line and the posterior point of the } \\
\text { superior temporal line }\end{array}$ \\
\hline 10 & Semi-landmark & S point & The superior point of the temporal fossa or the superior temporal line at the Frankfurt horizontal line \\
\hline 11 & Semi-landmark & ITSS point & The starting point of the inferior temporal septum on the temporal ridge \\
\hline 13-20 & Semi-landmark & ITSE point & The end point of the inferior temporal septum on the upper border of the zygomatic arch \\
\hline
\end{tabular}




\section{Statistical methods}

Statistical analysis was conducted using IBM SPSS Statistics for Windows version 27 (IBM Corp., Armonk, NY, USA). The average values of all the dimensions were analyzed. Data are presented as mean \pm standard deviation. Subsequently, the independent $\mathrm{t}$-test was performed to analyze differences between males and females. In addition, the intraclass correlation coefficient was used to evaluate intraobserver and interobserver reliability. Ten cadavers were chosen randomly, and two different distances were measured by two observers. The measurements were made twice at an interval of at least 2 weeks.

\section{Fig. 1. Illustrations of craniometric landmarks and semi-} landmarks

Lateral (A) and basal (B) views of the skull. The landmarks (red dots) and semi-landmarks (blue dots) are described in Table 1.

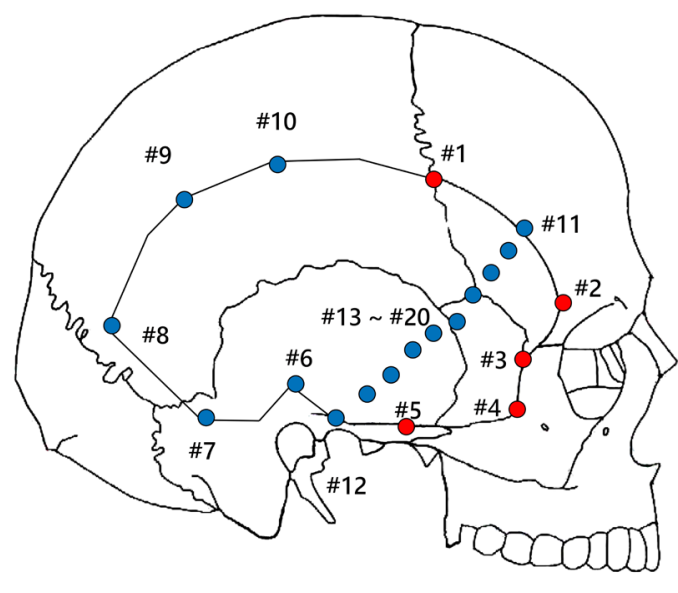

A

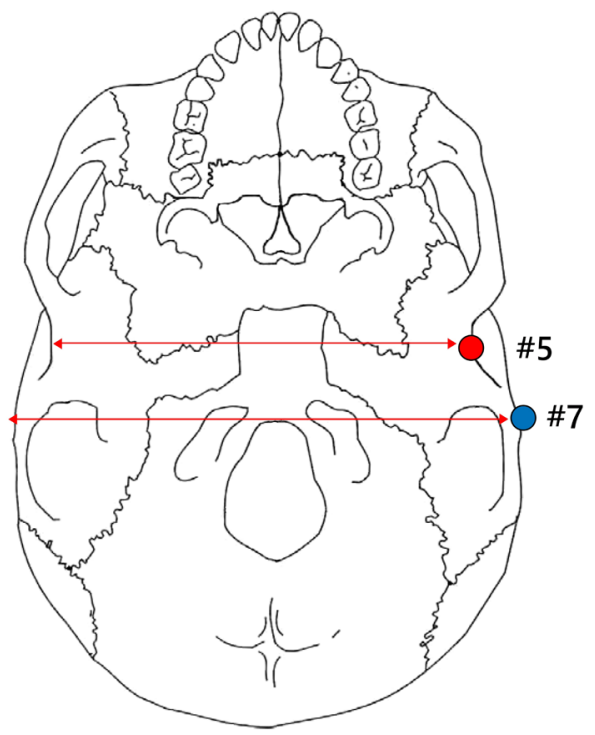

B

\section{RESULTS}

\section{Anatomical layers and boundaries of the temporal area}

After the dissection, the superficial temporal fascia (STF), innominate fascia (IF), DTF, superior temporal septum (STS), inferior temporal septum (ITS), and temporal ligamentous adhesion (TLA) were observed (Fig. 2, Supplemental Video 1).

The anatomical layers of the temporal area were identified as the skin (layer 1), subcutaneous fat tissue (layer 2), STF (layer 3), IF (layer 4), and DTF (layer 5). The most superficial layer

\section{Fig. 2. Schematic of boundaries of the temporal area}

$\mathrm{HB}$, harvestable boundary of the deep temporal fascia (blue broken line); ITS, inferior temporal septum; LOT, lateral orbital thickening; LTC, lower temporal compartment (green shaded area); STFP, superficial temporal fat pad; STS, superior temporal septum; SV, sentinel vein; TFN, temporal branch of the facial nerve; TLA, temporal ligamentous adhesion; UTC, upper temporal compartment (purple shaded area); ZTN, zygomaticotemporal nerve.

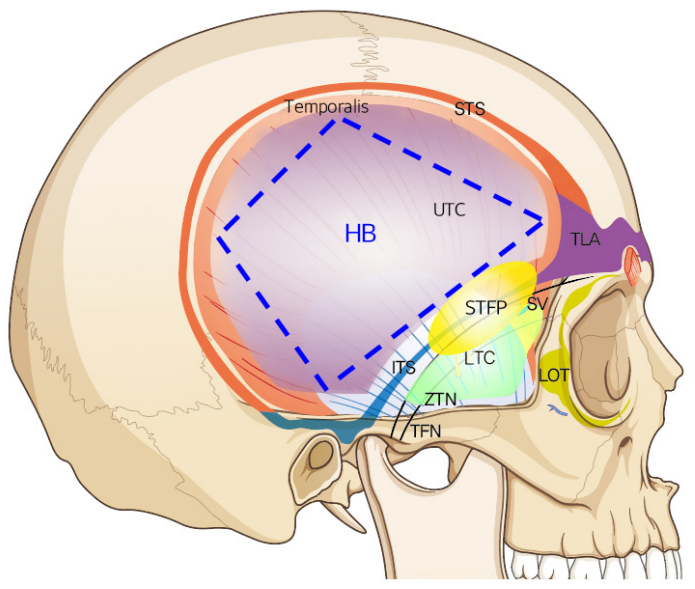

Fig. 3. Superficial temporal fascia

The superficial temporal artery and vein are visible in the superficial temporal fascia.

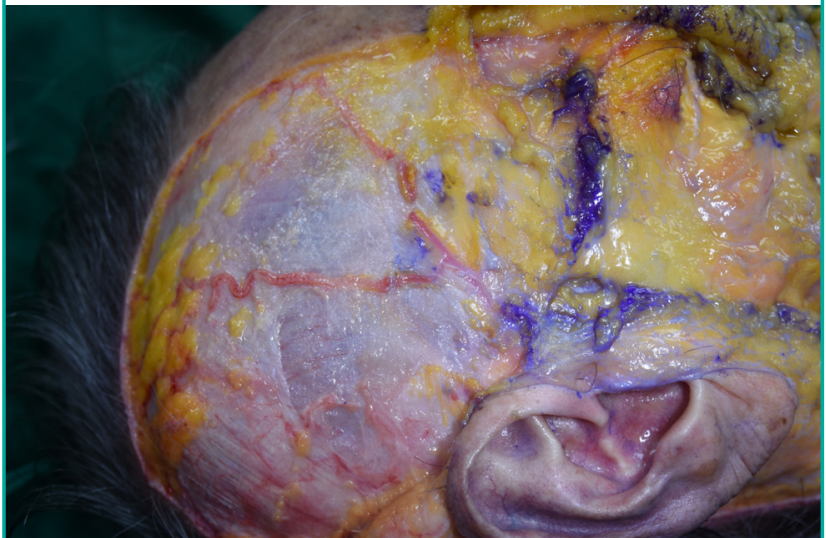


was the STF. The superficial temporal artery and vein ran through the STF and divided into the anterior and posterior branches $3 \mathrm{~cm}$ above the zygomatic arch (Fig. 3). The IF was a laminated loose areolar layer between the STF and the DTF

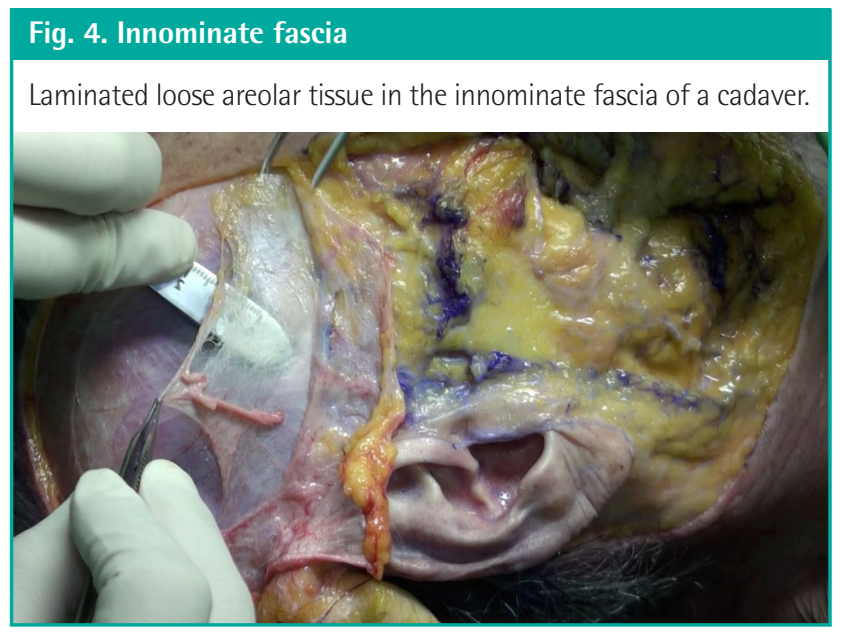

\section{Fig. 5. Superior temporal septum}

The superior temporal septum is densely attached to the periosteum.

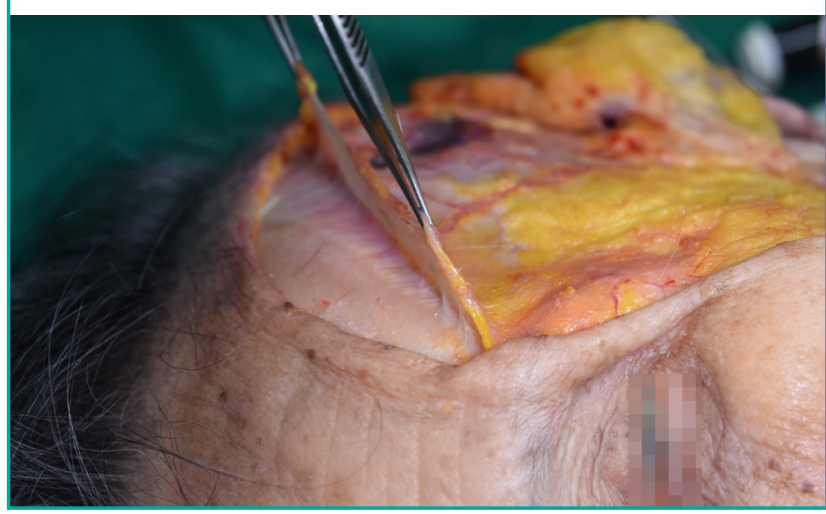

\section{Fig. 6. Inferior temporal septum}

A broad and undefined fibro-fatty structure of the inferior temporal septum, which is tightly connected to the deep temporal fascia enclosing the superficial temporal fat pad.

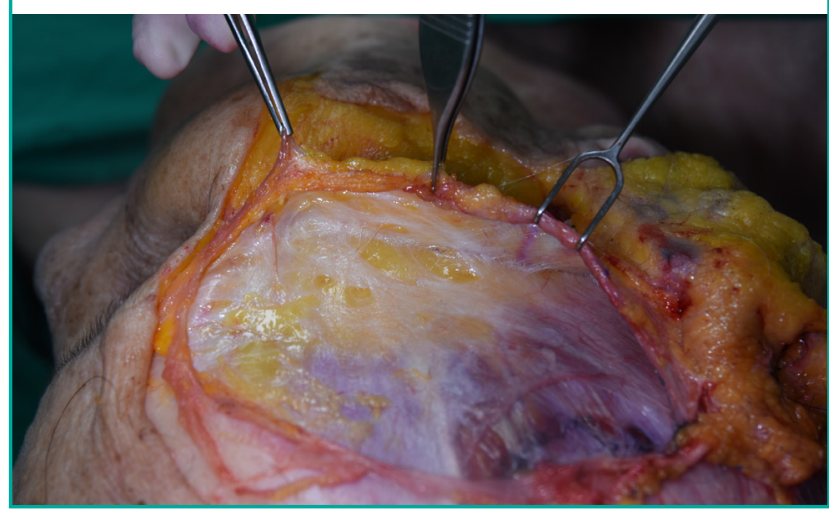

(Fig. 4). The STS was densely attached to the periosteum superoposteriorly and continued to the DTF (Fig. 5). The ITS was a broad and undefined fibro-fatty structure tightly connected with the DTF (Fig. 6). The superior and inferior temporal septa merged anteriorly into the TLA. The DTF was composed of superficial and deep laminae. The superficial temporal fat pad was enclosed between these two laminae under the ITS (Fig. 7).

The boundary of the ETC was the STS superoposteriorly, the TLA anteriorly, the upper border of the zygomatic arch anteroinferiorly, the IF laterally, and the DTF medially. The UTC and the LTC were divided by the ITS and the TLA. The UTC was easily dissected, and no major neurovascular structures were observed (Fig. 8). The boundary of the UTC was the STS superoposteriorly, the ITS anteroinferiorly, and the level of the upper border of the zygomatic arch posteroinferiorly. The LTC is anatomically complex due to its fibro-fatty components and neurovascular structures.

\section{Fig. 7. Superficial temporal fat pad}

The superficial temporal fat pad is located between the superficial and deep laminae of the deep temporal fascia.

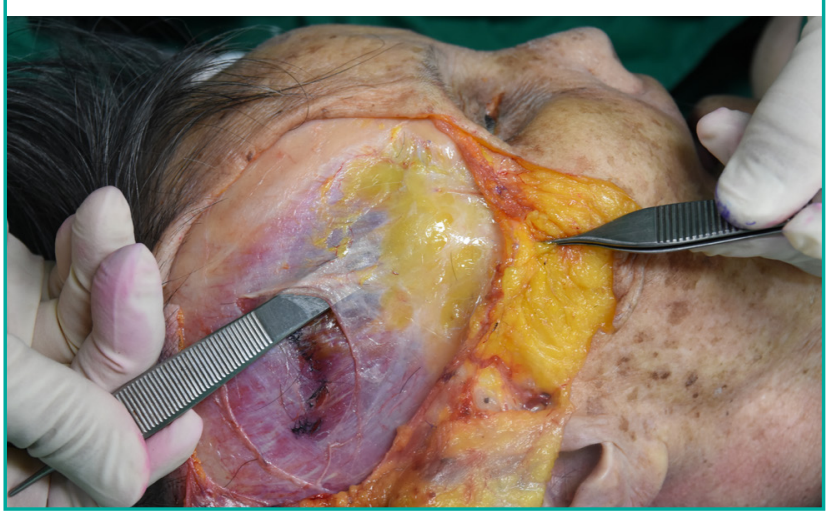

Fig. 8. Upper temporal compartment

The upper temporal compartment is located between the superior temporal septum and the inferior temporal septum and contains no major neurovascular structures.

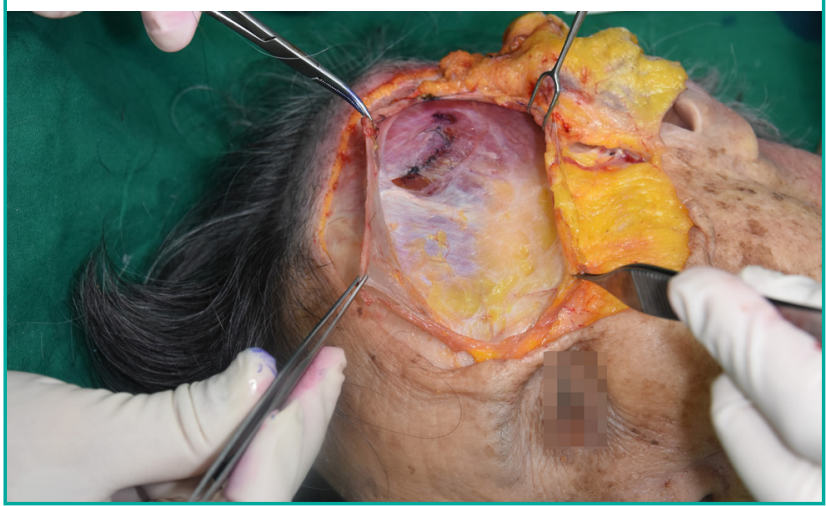




\section{Fig. 9. Landmarks and semi-landmarks}

Landmarks and the semi-landmarks were placed on the image to enable the calculation of the deep temporal fascia's dimensions.

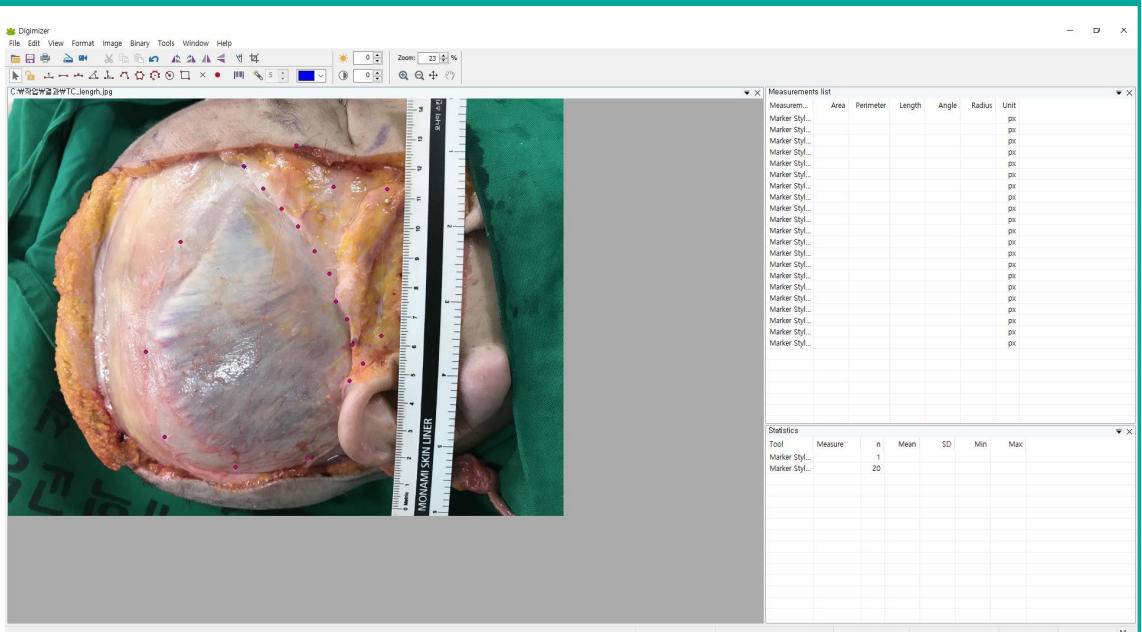

Fig. 10. Temporal compartment lengths

(A) Vertical (blue line) and horizontal (red line) lengths of the entire temporal compartment (blue shaded area). (B) Vertical (blue line) and horizontal (red line) lengths of the upper temporal compartment (yellow shaded area).
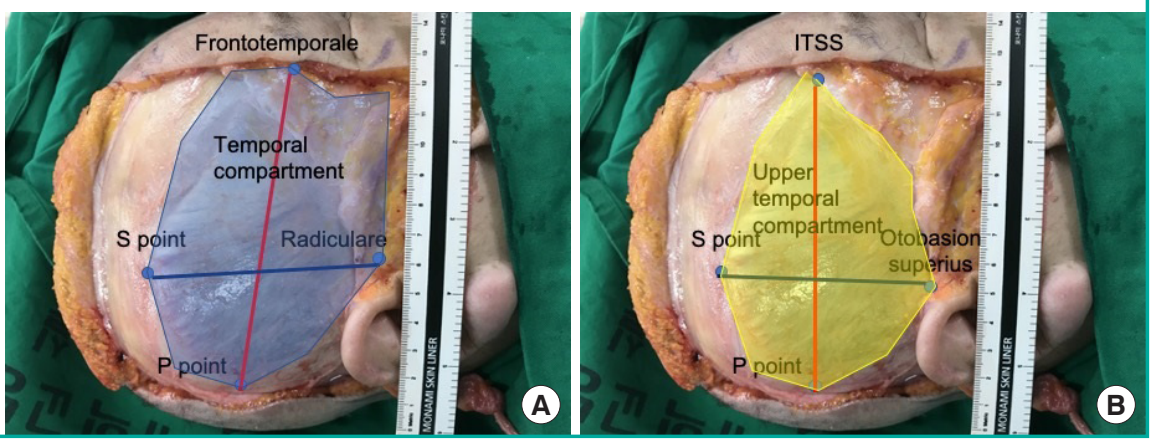

Table 2. Intraobserver and interobserver reliability assessment

\begin{tabular}{|c|c|c|c|c|}
\hline \multirow{2}{*}{ Evaluation } & \multicolumn{2}{|c|}{ Intraobserver, ICC (95\% CI) } & \multicolumn{2}{|c|}{ Interbserver, ICC (95\% CI) } \\
\hline & Observer 1 & Observer 2 & Session 1 & Session 2 \\
\hline Evaluation 1 & $0.993(0.971-0.998)$ & $0.983(0.933-0.996)$ & $0.975(0.905-0.994)$ & $0.971(0.890-0.993)$ \\
\hline Evaluation 2 & $0.982(0.931-0.996)$ & $0.989(0.955-0.997)$ & $0.948(0.807-0.987)$ & $0.943(0.790-0.986)$ \\
\hline
\end{tabular}

\section{Measurement of temporal compartment dimensions}

The intraobserver and interobserver agreement showed high consistency (interclass correlation coefficient $>0.75$ ) (Table 2). The five landmarks of the temporal fossa were placed on the digital images, and 15 semi-landmarks were added to represent the detailed curvature of the temporal compartments (Fig. 9). Eight of the 20 landmarks and semi-landmarks were used to measure the vertical and horizontal lengths of the temporal compartments (Fig. 10). The vertical and horizontal lengths of the ETC were $7.74 \pm 0.53 \mathrm{~cm}$ and $11.36 \pm 0.72 \mathrm{~cm}$, respectively. The vertical and horizontal lengths of the UTC were $6.41 \pm 0.67$ $\mathrm{cm}$ and $10.44 \pm 0.83 \mathrm{~cm}$, respectively (Table 3 ). To measure the surface area of the temporal compartments, the set of points

Table 3. Overall length of the temporal compartments
\begin{tabular}{|lrc|}
\hline \multirow{2}{*}{ Variable } & \multicolumn{2}{c|}{ Distance (cm) } \\
\cline { 2 - 3 } & Mean \pm SD & Median (range) \\
\hline TCVL & $7.74 \pm 0.53$ & $7.82(6.60-8.80)$ \\
TCHL & $11.36 \pm 0.72$ & $11.01(10.17-13.15)$ \\
UTCVL & $6.41 \pm 0.67$ & $6.40(5.27-7.50)$ \\
UTCHL & $10.44 \pm 0.83$ & $10.26(9.2-11.70)$ \\
\hline
\end{tabular}

TCVL, vertical length of the entire temporal compartment; TCHL, horizontal length of the entire temporal compartment; UTCVL, vertical length of the upper temporal compartment; UTCHL, horizontal length of the upper temporal compartment.

with landmarks and semi-landmarks was linked together on the images (Fig. 11). The average surface area of the ETC, UTC, 
Fig. 11. Temporal compartment surface area

The surface area of the compartments was computationally assessed by connecting the landmarks and the semi-landmarks described in Table 1.

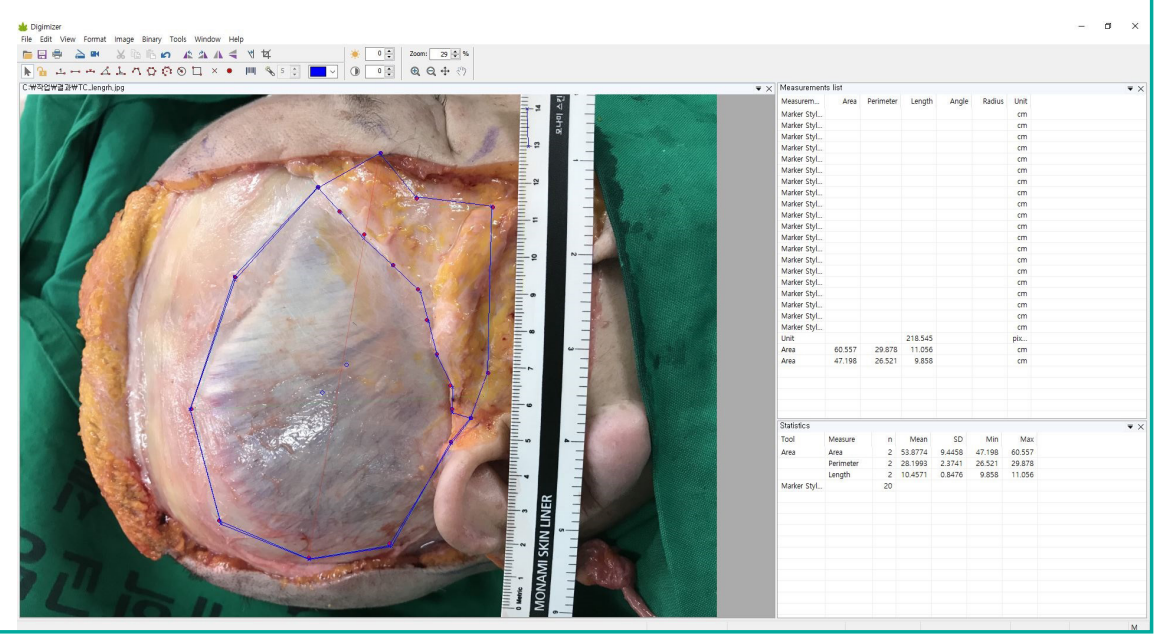

Table 4. Overall surface area of the temporal compartments

\begin{tabular}{|lcc|}
\hline \multirow{2}{*}{ Variable } & \multicolumn{2}{c|}{ Area $\left(\mathrm{cm}^{2}\right)$} \\
\cline { 2 - 3 } & Mean \pm SD & Median (range) \\
\hline ETC & $63.78 \pm 6.04$ & $64.72(52.43-73.42)$ \\
UTC & $48.52 \pm 5.65$ & $48.16(40.03-61.56)$ \\
LTC & $15.26 \pm 3.97$ & $15.02(9.21-23.96)$ \\
\hline
\end{tabular}

ETC, entire temporal compartment; UTC, upper temporal compartment; LTC, lower temporal compartment.

Table 5. Sex differences in the length of the temporal compartments

\begin{tabular}{|lrrrr|}
\hline \multirow{2}{*}{ Variable } & \multicolumn{2}{c}{ Distance $(\mathbf{c m})$} & \multirow{2}{*}{$\mathrm{t}$} & P-value $^{\mathrm{a})}$ \\
\cline { 2 - 3 } & \multicolumn{1}{c}{ Male } & \multicolumn{1}{c}{ Female } & & \\
\hline TCVL & $7.57 \pm 0.60$ & $7.86 \pm 0.45$ & 0.77 & 0.449 \\
TCHL & $11.49 \pm 0.89$ & $11.27 \pm 0.59$ & -1.456 & 0.158 \\
UTCVL & $6.42 \pm 0.59$ & $6.40 \pm 0.73$ & -0.168 & 0.868 \\
UTCHL & $10.40 \pm 0.92$ & $10.46 \pm 0.79$ & 0.058 & 0.954 \\
\hline
\end{tabular}

Values are presented as mean $\pm \mathrm{SD}$.

TCVL, vertical length of the entire temporal compartment; TCHL, horizontal length of the entire temporal compartment; UTCVL, vertical length of the upper temporal compartment; UTCHL, horizontal length of the upper temporal compartment. ${ }^{a}$ Statistically significant, $\mathrm{P}<0.05$.

and LTC were $63.78 \pm 6.04 \mathrm{~cm}^{2}, 48.52 \pm 5.65 \mathrm{~cm}^{2}$, and $15.26 \pm$ $3.97 \mathrm{~cm}^{2}$, respectively (Table 4). Although there were some differences in the results between the sexes, they were not statistically significant $(\mathrm{P}>0.05)$ (Tables 5, 6).

\section{DISCUSSION}

Harvesting the DTF without a sufficient anatomical understanding often causes postoperative complications such as bleeding, facial nerve paralysis, and temporal hollowness [15-
Table 6. Sex differences in the surface area of the temporal compartments

\begin{tabular}{|lccrc|}
\hline \multirow{2}{*}{ Variable } & \multicolumn{2}{c}{ Area $\left(\mathrm{cm}^{2}\right)$} & \multirow{2}{*}{$\mathrm{t}$} & P-value \\
\cline { 2 - 3 } & Male & Female & & \\
\hline ETC & $64.45 \pm 5.27$ & $63.31 \pm 6.64$ & 0.474 & 0.639 \\
UTC & $48.10 \pm 5.32$ & $48.80 \pm 6.02$ & -0.311 & 0.758 \\
LTC & $16.35 \pm 3.57$ & $14.51 \pm 4.17$ & 1.192 & 0.244 \\
\hline
\end{tabular}

Values are presented as mean $\pm \mathrm{SD}$.

ETC, entire temporal compartment; UTC, upper temporal compartment; LTC, lower temporal compartment.

a) Statistically significant, $P<0.05$.

19]. Previous studies reported the LTC contained all of the "atrisk" anatomical structures of the temporal area $[12,15,20]$. Moss et al. [12] reported that the sentinel vessels and both branches of the zygomaticotemporal nerve cross the LTC from deep to superficial on the inferomedial surface of the ITS. Huang et al. [20] reported that the anterior half of the LTC, where the sentinel vein and branches of the middle temporal vessels perforate the temporal fascia and travel into the subcutaneous tissue layer, is a "zone of caution." Vaca et al. [15] reported that preserving the superficial temporal fat pad on the ITS prevented postoperative temporal hollowness. We agree with the findings of those studies because of the anatomical complexity of the LTC.

In contrast, the layers and the ligamentous structures of the UTC were readily identified, as described in previous studies $[11,12]$. These structures are important for defining the boundary of the UTC and provide vital information about safe dissection. No major neurovascular structures were identified in the UTC. Therefore, the UTC is a surgically safe space through which one can easily harvest the DTF in rhinoplasty.

Several studies have reported harvestable boundaries of the 
Fig. 12. Deep temporal fascia contraction after harvest

Maximum harvestable boundary of the deep temporal fascia of a cadaver before (approximately $7 \times 10 \mathrm{~cm})(\mathrm{A})$ versus immediately after (approximately $5 \times 8 \mathrm{~cm})(\mathrm{B})$ harvest.

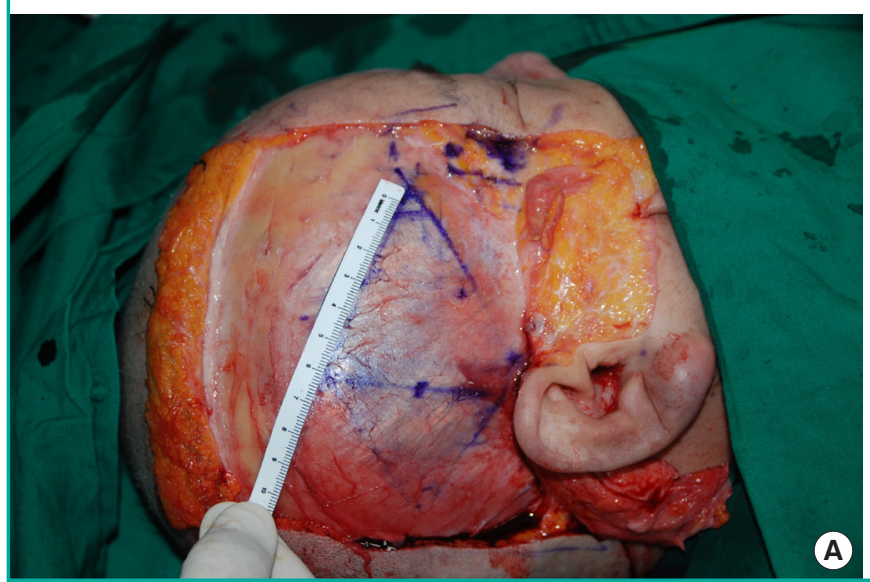

DTF and its maximum dimensions; however, its safe boundaries have not been described in detail [21-24]. Moran reported that each margin of the DTF ended in periosteal attachments at the frontal and parietal bones and at the upper edge of the zygomatic arch and that the maximum dimensions of the DTF were within $7 \times 7 \mathrm{~cm}$ [21]. Daniel and Palhazi [22] harvested the DTF with the resection superiorly to the periosteal junction, anteriorly to the DTF split, inferiorly toward the concha, and posteriorly as far back as possible. They usually harvested a $5 \times 5$ $\mathrm{cm}$ or larger piece of the DTF. Despite significant variations among individuals, Miller reported that a DTF sheet measuring approximately $4 \times 6 \mathrm{~cm}$ could be obtained with a thickness of approximately $1 \mathrm{~mm}$ [23]. Considering the risk of dissecting the ITC, the maximum harvestable boundary of the DTF is similar to that of the UTC, which had a mean value of $6.41 \times$ $10.44 \mathrm{~cm}$ in this study.

Rohrich et al. [24] incised the temporal fascia close to the attachments of the temporalis muscle to the skull periosteum superiorly and posteriorly and where it began to divide into the deep and superficial layers anteriorly and harvested the temporal fascia inferior to the level of the ear. They reported that the temporal fascia was approximately $8 \times 6 \mathrm{~cm}$; however, it contracted to approximately $5 \times 4 \mathrm{~cm}$ after the harvest. In addition, human and animal studies reported that the DTF contracts by $15 \%-$ $40 \%$ after harvest $[25,26]$. We also identified that the DTF measured $7 \times 10 \mathrm{~cm}$ before harvest and contracted to $5 \times 8 \mathrm{~cm}$ immediately after harvest (Fig. 12). Thus, a larger DTF area than needed should be harvested to compensate for the contraction.

Morphological differences in skulls between races and sex were reported previously [27]. It is generally accepted that dolichocephalic Caucasians have larger harvestable DTF areas than brachycephalic Asians owing to their anteroposteriorly long

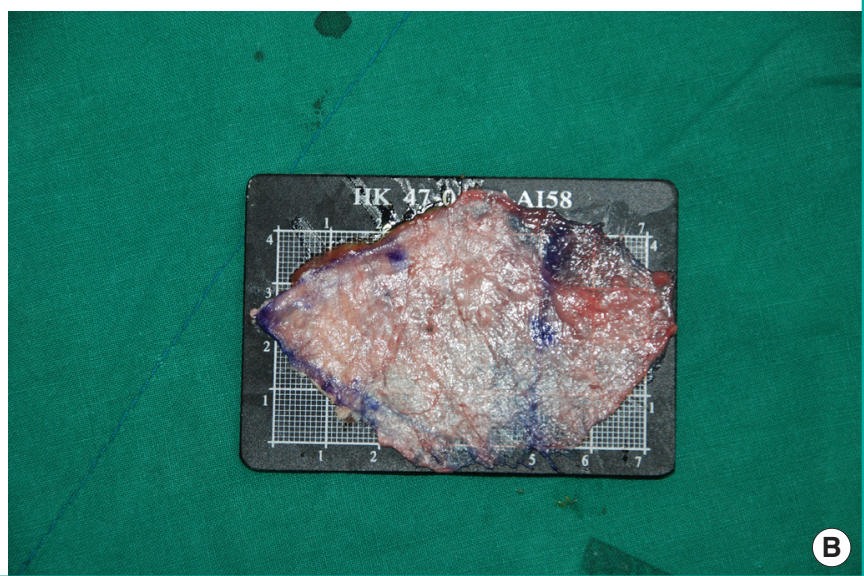

skull diameter. However, only a few studies have investigated the dimensions of the DTF, and most were conducted in Caucasians [21-24]. Moreover, no studies have compared the dimensions of the temporal area among different races. Prior to this study, we assumed that males had larger DTF areas than females considering the differences in their head sizes. However, we did not find statistically significant sex-based differences in any dimensions.

This study has some limitations. First, its sample size was small, and all samples were Korean. To reduce statistical errors and evaluate the differences among variable groups including race, sex, age, and height, sample size and diversity must be increased in further studies. Second, three-dimensional structures were measured by two-dimensional photogrammetric analysis, which always causes measurement errors regardless of intensity. Although two observers performed the measurements and the reliability assessment to avoid errors, this approach may not be sufficiently quantitative to ensure analytic precision.

In conclusion, use of the UTC enables sufficient DTF harvesting for clinical applications in rhinoplasty. A thorough understanding of the UTC boundary enables the easy and safe harvest of a sufficient amount of DTF.

\section{NOTES}

\section{Conflict of interest}

No potential conflict of interest relevant to this article was reported.

\section{Ethical approval}

The study was approved by the Institutional Review Board of Chungnam National University Hospital (IRB No. 2019-03- 
042) and performed in accordance with the principles of the Declaration of Helsinki.

\section{Patient consent}

The cadavers' guardian provided written informed consent for the publication and the use of images.

\section{Author contribution}

Conceptualization: D Chi, TK Kim. Data curation: S Kim. Formal analysis: JH Kim. Funding acquisition: SH Oh. Methodology: JH Kim. Project administration: JY Jeong, SH Oh. Visualization: C Shin. Writing - original draft: D Chi. Writing - review \& editing: $\mathrm{S}$ Kim, $\mathrm{SH}$ Oh.

\section{ORCID}

Daekwan Chi https://orcid.org/0000-0003-3903-4008 Jae-Hee Kim Taek-Kyun Kim Jae Yong Jeong Chungmin Shin Sunje Kim Sang-Ha Oh https://orcid.org/0000-0001-8022-4896 https://orcid.org/0000-0002-3213-9803 https://orcid.org/0000-0001-5654-4443 https://orcid.org/0000-0002-0613-649X https://orcid.org/0000-0001-7887-7521 https://orcid.org/0000-0003-3734-5005

\section{Supplementary material}

Supplemental Video 1. Temporal area dissection. Supplemental data can be found at: https://doi.org/10.5999/aps.2020.01165. v001.

\section{REFERENCES}

1. Nakajima H, Imanishi N, Minabe T. The arterial anatomy of the temporal region and the vascular basis of various temporal flaps. Br J Plast Surg 1995;48:439-50.

2. Han KH, Yeo HJ, Choi TH, et al. Rhinoplasty using various autogenous tissues. J Korean Soc Plast Reconstr Surg 2010; 37:37-45.

3. Jeong JY. Rebuilding nose: rhinoplasty for Asians. Uijeongbu: Medic Medicine; 2018.

4. Suh MK. Dorsal augmentation using autogenous tissue. In: Suh MK, Harijan A, Kim ST, editors. Atlas of Asian rhinoplasty. Singapore: Springer; 2018. p. 171-208.

5. Park SW, Kim JH, Choi CY, et al. Various applications of deep temporal fascia in rhinoplasty. Yonsei Med J 2015;56: 167-74.

6. Kim HK, Rhee SC. Augmentation rhinoplasty using a folded “pure” dermal graft. J Craniofac Surg 2013;24:1758-62.

7. Karaaltin MV, Batioglu-Karaaltin A, Orhan KS, et al. Autologous fascia lata graft for contour restoration and camouflage in tertiary rhinoplasty. J Craniofac Surg 2012;23:719-23.

8. Hong ST, Kim DW, Yoon ES, et al. Superficial mastoid fascia as an accessible donor for various augmentations in Asian rhinoplasty. J Plast Reconstr Aesthet Surg 2012;65:1035-40.

9. Guerrerosantos J. Temporoparietal free fascia grafts in rhinoplasty. Plast Reconstr Surg 1984;74:465-75.

10. Han JW, Sun H. Refinement of intraoral reverse temporalis transfer for facial palsy using a mandibular periosteum flap. Arch Plast Surg 2020;47:198-9.

11. O’Brien JX, Ashton MW, Rozen WM, et al. New perspectives on the surgical anatomy and nomenclature of the temporal region: literature review and dissection study. Plast Reconstr Surg 2013;131:510-22.

12. Moss CJ, Mendelson BC, Taylor GI. Surgical anatomy of the ligamentous attachments in the temple and periorbital regions. Plast Reconstr Surg 2000;105:1475-90.

13. Noback ML, Harvati K. Covariation in the human masticatory apparatus. Anat Rec (Hoboken) 2015;298:64-84.

14. Toro-Ibacache V, Zapata MuNoz V, O’higgins P. The predictability from skull morphology of temporalis and masseter muscle cross-sectional areas in humans. Anat Rec (Hoboken) 2015;298:1261-70.

15. Vaca EE, Purnell CA, Gosain AK, et al. Postoperative temporal hollowing: Is there a surgical approach that prevents this complication? A systematic review and anatomic illustration. J Plast Reconstr Aesthet Surg 2017;70:401-15.

16. Kim S, Matic DB. The anatomy of temporal hollowing: the superficial temporal fat pad. J Craniofac Surg 2005; 16:760-3.

17. ParkJA, Lee SH, Ha TJ, et al. Location of the split line of the deep temporal fascia when reducing a zygomatic arch fracture. J Plast Reconstr Aesthet Surg 2020;73:1130-4.

18. Lee JS, Kim JS, Lee JW, et al. Correction of microtia with constriction features using a superficial temporal fascial flap combined with a rib cartilage graft. Arch Plast Surg 2020;47: 317-23.

19. Cheon JH, Lee HC, Im GJ, et al. Safety and efficacy of transcutaneous bone conduction implant surgery for hearing improvement in microtia patients with bilateral hearing impairment. Arch Plast Surg 2019;46:525-34.

20. Huang RL, Xie Y, Wang W, et al. Anatomical study of temporal fat compartments and its clinical application for temporal fat grafting. Aesthet Surg J 2017;37:855-62.

21. Moran SL. Temporoparietal fascia flap. In: Wei FC, Mardini $S$, editors. Flaps and reconstructive surgery. Philadelphia: Elsevier; 2009.p. 159-73.

22. Daniel RK, Palhazi P. Rhinoplasty: an anatomical and clinical atlas. New York: Springer; 2018.

23. Miller TA. Temporalis fascia grafts for facial and nasal con- 
tour augmentation. Plast Reconstr Surg 1988;81:524-33.

24. Rohrich RJ, Adams WP, Ahmad J, et al. Dallas rhinoplasty: nasal surgery by the masters. Boca Raton: CRC Press; 2014.

25. Indorewala S. Dimensional stability of the free fascia grafts: an animal experiment. Laryngoscope 2002;112:727-30.
26. Indorewala S, Pagare R, Aboojiwala S, et al. Dimensional stability of the free fascia grafts: a human study. Laryngoscope 2004;114:543-7.

27. Jamieson A, Moenssens AA. Wiley encyclopedia of forensic science. New Jersey: Wiley; 2009. 